\title{
Heterogeneidade de Variância na Avaliação Genética de Reprodutores da Raça Pardo-Suíça no Brasil
}

\author{
Cláudio Vieira de Araújo1, Robledo de Almeida Torres ${ }^{2}$, Francisco Palma Rennó1, \\ José Carlos Pereira ${ }^{2}$, Rodolpho de Almeida Torres Filho ${ }^{1}$, Simone Inoe Araújo ${ }^{3}$, \\ Aldrin Vieira Pires ${ }^{2}$, Carla Aparecida Florentino Rodrigues ${ }^{1}$
}

\begin{abstract}
RESUMO - Informações de 6.842 lactações de 3.274 vacas da raça Pardo-Suíça, filhas de 71 reprodutores, distribuídas em 100 rebanhos, com parições entre 1980 a 1999, foram utilizadas para verificar a existência da heterogeneidade de variância entre rebanhos e o seu impacto na classificação de reprodutores. As produções de leite e gordura, ajustadas para 305 dias e para a idade adulta da vaca, foram utilizadas para classificar os rebanhos em níveis de alta e baixa produção. Utilizando-se um modelo animal que incluiu os efeitos fixos de rebanho, anoestação e grupo genético do animal, efeitos aleatórios de animal, efeito de ambiente permanente e ambiente temporário, estimaram-se componentes de variância considerando os rebanhos como uma única amostra e assumindo a produção de leite em cada nível de produção como característica diferente. Médias e componentes de variância foram maiores para o nível de alta produção, caracterizando a presença de heterogeneidade de variância entre os rebanhos. As estimativas de herdabilidade foram de 0,38 em ambos os níveis para a produção de leite e 0,39 e 0,32 para os níveis de alta e baixa produção, respectivamente. As correlações genéticas entre os níveis foram 0,85 e 0,79 para as produções de leite e gordura, respectivamente. Na avaliação genética de reprodutores, é importante considerar a variabilidade entre rebanhos, pois, se rebanhos mais variáveis contribuem com a maior parte dos animais, a seleção de reprodutor pelo desempenho de suas filhas, pode ser em função não apenas do seu potencial, mas também do ambiente no qual suas progênies expressam o fenótipo.
\end{abstract}

Palavras-chave: avaliação genética, bovinos leiteiros, parâmetros genéticos, produção de leite, produção de gordura

\section{Heterogeneity of Variance and Genetic Evaluation of Brown Swiss Cattle Breed in Brazil}

\begin{abstract}
Data from 6,842 lactations of 3,274 Brown Swiss cows, daughters of 71 sires, from 100 herds, calving from 1980 to 1999 were used to evaluate the effects of heterogeneity of variance on genetic evaluation of bulls. The milk and fat yields adjusted for 305 days of lactation and mature equivalent age were used to classify the herds among high and low production level. An animal model used to estimate variance component, included the fixed effect of herds, age-season of calving and grade of cows, random effect of animal, permanent environment and temporary environment. Variance components were estimated to milk (fat) yield in booth levels, considering the milk or fat yield in each production level as different trait. Estimates of heritability were $.38, .38, .39$ and .32 for milk and fat yields in high and low production levels, respectively. Genetic correlations between high and low production levels were .85 and .79 to milk and fat yields, respectively. In genetic evaluation programs, it is important to consider differences in variability among herds, otherwise, under selection, the most variables herds would contribute with the majority of the animals, and the genetic evaluations of the animals could be more a function not only from its genetic potential, but also from its environment where the progenies expressed their traits.
\end{abstract}

Key Words: dairy cattle, genetic evaluation, genetic parameters, milk yield, fat yield

\section{Introdução}

Variâncias heterogêneas entre rebanhos e aumento das variâncias, de acordo com o incremento dos níveis de produções dos rebanhos, regiões ou países, têm sido verificados por vários autores, como Hill et al. (1983), Boldman \& Freeman (1990), Dong \& Mao (1990), Stanton et al. (1991), Costa (1998), Torres (1998) e Araújo (2000), com o objetivo de verificar o efeito da heterogeneidade de variância da produção de leite entre rebanhos sobre a avaliação genética e a classificação de animais.

Quando a heterogeneidade de variância é desprezada, se presente, a produção das filhas de determinado reprodutor será ponderada na proporção dos desviospadrão dos rebanhos em que essas filhas foram criadas. O resultado é que as produções das filhas oriundas de rebanhos com maiores variâncias influenciarão mais a avaliação de reprodutores que a de filhas oriundas de rebanhos com menores variâncias (Torres, 1998).

\footnotetext{
1 Estudante de Doutorado da UFV. E.mail: araujocv@bol.com.br

2 Professor do Departamento de Zootecnia da UFV. E.mail: rtorres@mail.ufv.br

3 Engenheira-Agrônoma. E.mail: znoearaújo@bol.com.br
} 
Short et al. (1990), trabalhando com lactações de 299.441 vacas em rebanhos estratificados de acordo com o desvio-padrão fenotípico da produção de leite ajustada para idade adulta, em três níveis (baixo, médio e alto), observaram que os componentes de variância de reprodutor e residual elevaram-se com o aumento do desvio-padrão fenotípico. As herdabilidades para produção de leite na primeira lactação foram $0,15,0,26$ e 0,23 , para as classes de desvio-padrão, baixo, médio e alto, respectivamente.

Boldman \& Freeman (1990) estudaram dados de 121.136 primeiras lactações e 91.206 lactações subseqüentes de vacas Holandesas. As variâncias genéticas aditiva e residual para todas as lactações foram estimadas em três níveis de produção de rebanho. Cada grupo rebanho-ano-estação foi designado para os níveis de produção com base na média da produção de todas as vacas que produziram no mesmo rebanho-ano. Os componentes de variância aumentaram com o nível de produção.

Lee \& Park (1994) trabalharam com dados de produção de leite de rebanhos estratificados em grupos de baixa, média e alta produção, com base nas médias de produção das classes de rebanho-ano. Os autores evidenciaram presença da heterogeneidade nos três níveis de produção de leite.

Torres (1998), por meio de lactações de vacas da raça Holandesa no Brasil, estratificou os rebanhos em classes de desvio-padrão fenotípico alta, média e baixa. As médias e os componentes de variância genética elevaram com o aumento do desvio-padrão médio da classe, demonstrando maior expressão do potencial genético dos animais nos rebanhos de maior desvio-padrão fenotípico.

Costa (1999) utilizou registros de produção de leite e gordura de vacas de primeira lactação da raça Holandesa com o objetivo de verificar a heterogeneidade de variância entre rebanhos. Com base no desvio-padrão fenotípico da produção de leite por classes de rebanho-ano, os rebanhos foram divididos em alto e baixo desvio-padrão. Os componentes de variância de reprodutor e de resíduo para as produções foram maiores na classe de alto desviopadrão fenotípico.

Com base no desvio-padrão fenotípico da produção de leite, Araújo (2000) estratificou os rebanhos em três classes de desvio-padrão; alto, médio e baixo. Médias de produção de leite, componentes de variância e herdabilidades aumentaram da classe de baixo para alto desvio-padrão fenotípico, caracterizando a heterogeneidade de variância entre os rebanhos.

Este estudo foi conduzido com o objetivo de verificar a existência da heterogeneidade de variância entre rebanhos e seu impacto na avaliação genética e classificação de reprodutores da raça Pardo-Suíça no Brasil.

\section{Material e Métodos}

Foram utilizados registros de 6.842 lactações provenientes da Associação Brasileira de Criadores de Gado Pardo-Suíço do Brasil. Os registros referem-se a lactações de 3.274 vacas da raça PardoSuíça, filhas de 71 reprodutores, distribuídas em 100 rebanhos, com parições nos anos de 1980 a 1999. As lactações foram ajustadas para 305 dias de lactação e para idade adulta da vaca, correspondente aos 72 meses de idade, utilizando fatores estimados por Rennó (2001). Foram calculadas médias referentes às produções de leite e gordura para cada rebanho. Os rebanhos foram classificados em níveis alto e baixo de produção. O critério utilizado para estratificação dos rebanhos em dois níveis foi a distância Euclidiana, que é uma medida de dissimilaridade, expressa por Johnson \& Wichern, (1998) como:

$$
\mathrm{d}_{(\mathrm{A}, \mathrm{B})}=\sqrt{\left(\overline{\mathrm{X}}_{\mathrm{PL}(\mathrm{A})}-\overline{\mathrm{X}}_{\mathrm{PL}(\mathrm{B})}\right)^{2}+\left(\overline{\mathrm{X}}_{\mathrm{PG}(\mathrm{A})}-\overline{\mathrm{X}}_{\mathrm{PG}(\mathrm{B})}\right)^{2}},
$$

em que: $d_{(A, B)}$ é a distância Euclidiana entre os rebanhos $\mathrm{A}$ e B $\overline{\mathrm{X}}_{\mathrm{PL}(\mathrm{A})}, \overline{\mathrm{X}}_{\mathrm{PG}(\mathrm{A})}$ e $\overline{\mathrm{X}}_{\mathrm{PL}(\mathrm{B})}$ e $\overline{\mathrm{X}}_{\mathrm{PG}(\mathrm{B})}$, as produções médias de leite e gordura nos rebanhos $\mathrm{A}$ e B, respectivamente. O rebanho detentor de maior média para a produção de leite foi tomado como base para a formação do nível de alta produção, no qual foram incluídos $50 \%$ dos rebanhos com menores valores para a distância Euclidiana para o rebanhobase (rebanho mais similar em relação a maiores produções de leite e gordura). Os 50\% dos rebanhos restantes, correspondentes aos rebanhos menos similares, isto é, aqueles que apresentaram maiores distâncias Euclidianas em relação ao rebanho-base, foram agrupados no nível de baixa produção.

Após os rebanhos serem classificados nos níveis alto e baixo de produção, os dados foram editados de forma que os reprodutores possuíssem filhas nos dois níveis de produção e, ainda, pelo menos duas filhas em cada nível de produção, o que resultou em 72 reprodutores em comum nos dois níveis de produção. Os meses de parto das vacas foram agrupados em quatro épocas de parto: 1) janeiro a março, 2) abril a 
junho, 3) julho a setembro e 4) outubro a dezembro.

Com referência à informação sobre ascendência e origem das vacas, classificaram-se os animais em Grupo 1, animais puros de origem, Grupo 2, animais puros por cruzamento e Grupo 3, animais puros de origem importada.

As estimativas dos componentes de (co)variâncias e dos parâmetros genéticos foram obtidas com o programa MTDFREML (Multiple Trait Derivative Free Restricted Maximum Likelihood), descrito por Boldman et al. (1995), que utilizam a máxima verossimilhança restrita livre de derivação. $\mathrm{O}$ critério de convergência adotado foi a variância dos valores do simplex de, no mínimo, 10-9.

Por meio de modelo unicaracter para produção de leite e gordura, distintamente, que incluiu os efeitos fixos de rebanho, ano-estação e grupo genético do animal, efeitos aleatórios de animal, efeito de ambiente permanente e ambiente temporário, estimaram-se componentes de variância considerando as produções de leite ou gordura em todos os rebanhos conjuntamente. Um modelo multicaracterístico com os mesmos efeitos do modelo anterior e que descreveu o desempenho das vacas, considerando a produção de leite (ou gordura) em cada nível de produção como uma característica distinta, foi usado para estimar os componentes de variâncias para as produções de leite e gordura, descrito como: $y=X \beta+Z a+Z p+e$, em que:

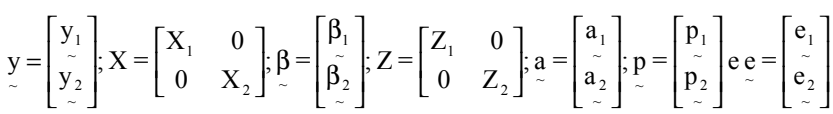
sendo y o vetor referente à $\mathrm{n}$ observação da produção de leite (ou gordura) ajustada para 305 dias de lactação e para idade adulta da vaca correspondente às vacas no nível de produção $\mathrm{i} ; \mathrm{X}_{\mathrm{i}}$ a matriz de incidência de efeitos fixos de rebanho, ano-estação de parto e grupo genético de animal no nível de produção $i ; \beta_{i}$ o vetor de efeitos fixos no nível de produção $i ; Z_{i}$ a matriz de incidência do efeito aleatório; e $a_{i}, p_{i}$ e e $e_{i}$ são vetores de efeitos aleatórios genético aditivo, ambiente permanente e efeito temporário de ambiente, respectivamente. As pressuposições assumidas acerca da distribuição dos vetores $\mathrm{y}, \beta, \mathrm{a}, \mathrm{p}$ e e, sob distribuição multivariada, podem ser ${ }^{2}$ ẽscritas como

R. Bras. Zootec., v.31, n.3, p.1343-1349, 2002 (suplemento)

$$
\left[\begin{array}{c}
\mathrm{y} \\
\tilde{\mathrm{a}} \\
\tilde{\mathrm{p}} \\
\tilde{\mathrm{e}} \\
\sim
\end{array}\right] \cong\left\{\left[\begin{array}{c}
\mathrm{X} \beta \\
\phi \\
\phi \\
\phi
\end{array}\right],\left[\begin{array}{cccc}
\mathrm{ZGZ}+\mathrm{ZPZ}^{\prime}+\mathrm{R} & \mathrm{ZG} & \mathrm{ZP} & \mathrm{R} \\
\mathrm{GZ} & \mathrm{G} & \phi & \phi \\
\mathrm{PZ} & \phi & \mathrm{P} & \phi \\
\mathrm{R} & \phi & \phi & \mathrm{R}
\end{array}\right]\right\},
$$

em que:

$$
\begin{aligned}
& G=A \otimes G_{0}, \\
& \mathrm{P}=\mathrm{I}_{N} \otimes P_{0} \mathrm{e}, \\
& R=I_{n} \otimes R_{0}
\end{aligned}
$$

sendo A a matriz de numeradores dos coeficientes de parentesco entre indivíduos, de ordem igual ao número de indivíduos $(\mathrm{N}) ; \mathrm{G}_{\mathrm{O}}$ a matriz de (co)variância genética aditiva entre as características; IN uma matriz identidade, de ordem $\mathrm{N} ; \mathrm{P}_{\mathrm{O}}$ a matriz de variância referente ao efeito de ambiente permanente de animal; In matriz identidade, de ordem igual ao número total de observações (n); e $\mathrm{R}_{0}$ matriz de variância residual entre as características.

As equações de modelo misto, assumindo as produções em cada nível como características diferentes, são descritas como

$$
\left[\begin{array}{ccc}
X^{\prime} R^{-1} X & X^{\prime} R^{-1} Z & X^{\prime} R^{-1} Z \\
Z^{\prime} R^{-1} X & Z^{\prime} R^{-1} Z+G^{-1} & Z^{\prime} R^{-1} Z \\
Z^{\prime} R^{-1} X & Z^{\prime} R^{-1} Z & Z^{\prime} R^{-1} Z+P^{-1}
\end{array}\right]\left[\begin{array}{c}
\beta^{0} \\
\hat{\hat{a}} \\
\hat{\hat{p}}
\end{array}\right]=\left[\begin{array}{cc}
X^{\prime} R^{-1} & y \\
Z^{\prime} R^{-1} & y \\
Z^{\prime} R^{-1} & y
\end{array}\right],
$$

cuja solução é obtida da seguinte forma:

$\hat{\beta}^{0}=\left[X^{\prime} R^{-1} X-X^{\prime} R^{-1} Z\left(Z^{\prime} R^{-1} Z+G^{-1}\right)^{-1} Z^{\prime} R^{-1} X\right]^{-}\left[X^{\prime} R^{-1} y-X^{\prime} R^{-1} Z\left(Z^{\prime} R^{-1} Z+G^{-1}\right)^{-1} Z^{\prime} R^{-1} y\right]$,

$$
\hat{a}=\left(Z^{\prime} R^{-1} Z+G^{-1}\right)^{-1} Z^{\prime} R^{-1}(y-X \beta),
$$

Com base nos componentes de co(variâncias) estimados, calculou-se a eficiência da resposta da seleção indireta das produções no nível de baixa produção, em resposta à seleção das produções no nível de alta produção, em relação à resposta direta para as produções no nível de baixa produção, como: $\frac{\mathrm{RC}_{\mathrm{Y}, \mathrm{X}}}{\mathrm{R}_{\mathrm{y}}^{2}}=\mathrm{r}_{\mathrm{axy}} \frac{\mathrm{i}_{\mathrm{x}}}{\mathrm{i}_{\mathrm{y}}} \frac{\mathrm{h}_{\mathrm{x}}}{\mathrm{h}_{\mathrm{y}}}$, em que $\mathrm{RC}_{\mathrm{Y}, \mathrm{X}}$ é a razão da resposta correlacionada das produções no nível de baixa produção (y) em resposta à seleção direta das produções no nível de alta produção $(\mathrm{x}) ; \mathrm{R}_{\mathrm{y}}^{2}$, a resposta da seleção direta para as produções no nível de baixa produção; $r_{\text {axy }}$ é a correlação genética entre as produções nos níveis de alta e baixa produção e $\mathrm{i}_{\mathrm{x}}, \mathrm{i}_{\mathrm{y}}, \mathrm{h}_{\mathrm{x}}$ e $\mathrm{h}_{\mathrm{y}}$ as intensidades de seleção e raízes das estimativas de herdabilidade nos níveis de alta e baixa produção, respectivamente. 
Com base nos valores genéticos preditos para os reprodutores em ambos os níveis de produção, calcularam-se as correlações de Pearson e Spearman, com o objetivo de verificar possíveis alterações na classificação dos reprodutores nos diferentes níveis de produção.

\section{Resultados e Discussão}

As médias para as produções de leite e gordura, considerando os rebanhos conjuntamente (análise geral), foram 5941,43 1661,66 kg e 221,28 61,13 kg, respectivamente. As distribuições do número de animais, lactações, rebanhos e reprodutores em cada nível de produção e em análise geral, após a edição dos dados, podem ser observadas na Tabela 1 .

As distribuições dos animais e das lactações em cada grupo genético para cada nível de produção, e em análise geral, podem ser observadas na Tabela 2 .

Tabela 1 - Número de animais, de lactações, de rebanhos e de reprodutores, em cada nível de produção, e considerando todos rebanhos

Table 1 - Number of animals, lactations, herds and sires, in each production level, and considering all data

\begin{tabular}{lccc}
\hline & \multicolumn{2}{c}{$\begin{array}{c}\text { Nível de produção } \\
\text { Production level }\end{array}$} & $\begin{array}{c}\text { Geral } \\
\text { All data }\end{array}$ \\
\cline { 2 - 3 } & $\begin{array}{c}\text { Alto } \\
\text { High }\end{array}$ & $\begin{array}{c}\text { Baixo } \\
\text { Low }\end{array}$ & \\
\hline $\begin{array}{l}\mathrm{N}^{0} \text { de animais } \\
\text { Number of animals }\end{array}$ & 2033 & 1241 & 3274 \\
$\begin{array}{l}\mathrm{N}^{0} \text { de lactações } \\
\text { Number of lactations }\end{array}$ & 4260 & 2582 & 6842 \\
$\mathrm{~N}^{\mathrm{O}}$ de rebanhos & 55 & 45 & 100 \\
$\begin{array}{l}\text { Number of herds } \\
\mathrm{N}^{0} \text { de reprodutores } \\
\text { Number of sires }\end{array}$ & 72 & 72 & 72 \\
\hline
\end{tabular}

Os componentes de variância e parâmetros genéticos para as produções, em cada nível de produção, podem ser observados na Tabela 3. Os componentes de variância genética aditiva e de ambiente temporário (residual) foram menores no nível de baixa produção que no nível de alta produção em ambas as características. A estimativa do componente de variância referente ao efeito permanente de ambiente foi próxima a zero em todas as situações, revelando que este efeito pouco contribuiu na variabilidade das produções. Este resultado revela baixa variância das produções mensuradas em diferentes lactações entre indivíduos, que são atribuídas ao ambiente.

Apesar da maior estimativa de componente de variância genética aditiva para a produção de leite ajustada para 305 dias e para a idade adulta da vaca, este aumento foi proporcional na variância residual, resultando na mesma estimativa de herdabilidade em ambos níveis de produção. Por outro lado, observa-se maior estimativa de herdabilidade para a produção de gordura, no nível de alta produção de gordura, em conseqüência da maior variabilidade genética aditiva neste nível, quando comparado com o nível de baixa produção.

Os altos valores das correlações genéticas entre as produções nos níveis de alta e baixa produção (Tabela 3) revelam que seria esperado que os animais fossem classificados de maneira similar em ambos os níveis de produção. Boldman \& Freeman (1990), Torres et. al (2000), Costa (1999) e Araújo (2000) encontraram resultados de correlação genética maiores que 0,90 , entre a mesma característica em classes de grupos de rebanhos diferentes.

Com base nos valores estimados dos componentes de variâncias e covariâncias genéticas, foram calculadas as eficiências das respostas esperadas à

Tabela 2 - Número de animais e de lactações em cada grupo genético, para cada nível de produção, e considerando todos rebanhos

Table 2 - Number of animals and of lactations in each genetic group, for each production level, and considering all data

\begin{tabular}{|c|c|c|c|c|}
\hline & & \multicolumn{2}{|c|}{$\begin{array}{c}\text { Nível de produção } \\
\text { Production level }\end{array}$} & \multirow[t]{2}{*}{$\begin{array}{l}\text { Geral } \\
\text { All data }\end{array}$} \\
\hline & & $\begin{array}{l}\text { Alto } \\
\text { High }\end{array}$ & $\begin{array}{l}\text { Baixo } \\
\text { Low }\end{array}$ & \\
\hline Grupo genético 1 & $\mathrm{~N}^{0}$ de animais & 1375 & 936 & 2311 \\
\hline Genetic group 1 & $\mathrm{~N}^{\mathrm{o}}$ de lactações & 2722 & 1997 & 4719 \\
\hline Grupo genético 2 & $\mathrm{~N}^{\mathrm{O}}$ de animais & 309 & 221 & 530 \\
\hline Genetic group 2 & $\mathrm{~N}^{0}$ de lactações & 768 & 389 & 1157 \\
\hline Grupo genético 3 & $\mathrm{~N}^{\circ}$ de animais & 349 & 84 & 433 \\
\hline Genetic group 3 & $\mathrm{~N}^{0}$ de lactações & 770 & 196 & 996 \\
\hline
\end{tabular}

\section{R. Bras. Zootec., v.31, n.3, p.1343-1349, 2002 (suplemento)}


Tabela 3 - Média, desvio-padrão (DP), número de lactações (N), componentes de variância, herdabilidades e correlações genéticas para as produções de leite e gordura ajustadas para 305 dias de lactação e para idade adulta da vaca, nos níveis de alta e baixa produção

Table 3 - Average, standard deviation (DP), number of lactations (N), variance components, heritability and genetic correlation to milk and fat yields adjusteds for 305 days and mature equivalent age of the cow to high and low production levels

\begin{tabular}{|c|c|c|c|c|}
\hline \multirow[b]{2}{*}{ Item } & \multicolumn{2}{|c|}{$\begin{array}{l}\text { Produção de leite } \\
\text { Milkyield }\end{array}$} & \multicolumn{2}{|c|}{$\begin{array}{c}\text { Produção de gordura } \\
\text { Fat yield }\end{array}$} \\
\hline & $\begin{array}{l}\text { Alto } \\
\text { High }\end{array}$ & $\begin{array}{l}\text { Baixo } \\
\text { Low }\end{array}$ & $\begin{array}{l}\text { Alto } \\
\text { high }\end{array}$ & $\begin{array}{l}\text { Baixo } \\
\text { Low }\end{array}$ \\
\hline $\begin{array}{l}\text { Média, } \mathrm{kg} \\
\text { Mean }\end{array}$ & 6541,90 & 4951,78 & 240,77 & 189,12 \\
\hline $\mathrm{DP}$ & 1578,00 & 1278,87 & 60,32 & 42,30 \\
\hline $\begin{array}{l}\mathrm{N} \\
(\text { Co }) \text { variância genética aditiva }\left(\mathrm{kg}^{2}\right)\end{array}$ & 4260 & 2582 & 4260 & 2582 \\
\hline $\begin{array}{l}\text { Genetic additive (co)variance }\left(\mathrm{kg}^{2}\right) \\
\text { Alto } \\
\text { Hioh }\end{array}$ & 699611,50 & 490946,43 & 1078,19 & 610,66 \\
\hline $\begin{array}{l}\text { Baixo } \\
\text { Low }\end{array}$ & & 472749,58 & & 562,23 \\
\hline $\begin{array}{l}\text { Variância de ambiente permanente }\left(\mathrm{kg}^{2}\right) \\
\text { Environment permanent variance }\left(\mathrm{kg}^{2}\right)\end{array}$ & & & & \\
\hline $\begin{array}{l}\text { Variância de ambiente temporário }\left(\mathrm{kg}^{2}\right) \\
\text { Environment temporarv variance }\left(\mathrm{kg}^{2}\right)\end{array}$ & $2,15 \times 10^{-2}$ & $5,62 \times 10^{-2}$ & $2,35 \times 10^{-2}$ & $2,73 \times 10^{-3}$ \\
\hline $\begin{array}{l}\text { Herdabilidades e correlações genéticas } \\
\text { Heritability and genetic correlations }\end{array}$ & 1138587,31 & 780721,23 & 1694,35 & 1205,15 \\
\hline $\begin{array}{l}\text { Alto } \\
\text { High }\end{array}$ & 0,38 & 0,85 & 0,39 & 0,79 \\
\hline $\begin{array}{l}\text { Baixo } \\
\text { Low }\end{array}$ & & 0,38 & & 0,32 \\
\hline
\end{tabular}

seleção no nível de baixa produção pela seleção no nível de alta produção, em relação à resposta direta no nível de baixa produção, admitindo a mesma intensidade de seleção em ambos os níveis. Com base nesses resultados, a resposta da eficiência resultaria em 0,85 e $0,87 \%$, para as produções de leite e gordura, respectivamente. Verifica-se, portanto, que a transferência do material genético selecionado no nível de alta produção conduz a menores respostas nos desempenhos dos reprodutores, quando submetidos aos rebanhos de menores produções, em relação à situação em que a seleção dos animais no nível de baixa produção é praticada diretamente neste nível. Stanton et al. (1991), Torres (1998) e Costa (1999) verificaram menores respostas correlacionadas em rebanhos de menor desvio-padrão fenotípico por unidade de ganho nos rebanhos de alto desvio-padrão fenotípico.

Médias, desvios-padrão, valores mínimos e máximos de valores genéticos dos reprodutores para as produções de leite e de gordura, em cada nível de produção e em análise geral, são apresentados na Tabela 4. As médias e os desvios-padrão foram maiores para o nível de alta produção em ambas as características. Os valores genéticos e amplitudes foram maiores no nível de alta produção. Desde que os reprodutores possuem maior número de filhas em rebanhos de alta produção, estes possivelmente teriam maiores valores genéticos, em decorrência da maior concentração de suas progênies em rebanhos de maior variabilidade - cerca de $62 \%$. No caso de reprodutores que têm seus valores genéticos obtidos com base no desempenho de suas progênies, a resposta à seleção seria também em função do número de filhas utilizadas na avaliação do reprodutor, bem como dos ambientes a que elas seriam submetidas e da distribuição dessas filhas nos vários ambientes. Portanto, é importante considerar as condições de meio ambiente a que serão submetidos os seus descendentes, uma vez que a resposta em termos de produção está relacionada com essas condições. 
Tabela 4 - Número médio de filhas, médias, desvios-padrão, valores mínimos e máximos de valores genéticos dos reprodutores em cada nível de produção e em análise geral

Table 4 - Average number of daughters, average, standard-deviation, minimum and maximum values of genetic values of the reproducers in each production level and in general analysis

\begin{tabular}{|c|c|c|c|c|}
\hline $\begin{array}{l}\text { Nível de produção } \\
\text { Production of level }\end{array}$ & $\begin{array}{l}\mathrm{N}^{\mathrm{O}} \text { médio de filhas } \\
\text { Number of daughters }\end{array}$ & $\bar{X} \pm D P$ & $\begin{array}{l}\text { Mínimo } \\
\text { Minimum }\end{array}$ & $\begin{array}{l}\text { Máximo } \\
\text { Maximum }\end{array}$ \\
\hline \multicolumn{5}{|c|}{$\begin{array}{l}\text { Produção de leite }(\mathrm{kg}) \\
\text { Milkyield } \\
\end{array}$} \\
\hline Alto & 28,23 & $79,67 \pm 510,70$ & -1707 & 1298 \\
\hline High & & & & \\
\hline Baixo & 17,24 & $50,98 \pm 443,60$ & -1344 & 1045 \\
\hline $\begin{array}{l}\text { Geral } \\
\text { Overall }\end{array}$ & 45,47 & $69,05 \pm 547,18$ & -1550 & 1234 \\
\hline \multicolumn{5}{|c|}{$\begin{array}{c}\text { Produção de gordura (kg) } \\
\text { Fat yield }\end{array}$} \\
\hline $\begin{array}{l}\text { Alto } \\
\text { High }\end{array}$ & 28,23 & $1,17 \pm 18,99$ & $-70,91$ & 50,10 \\
\hline $\begin{array}{l}\text { Baixo } \\
\text { Low }\end{array}$ & 17,24 & $0,22 \pm 15,47$ & $-45,88$ & 49,45 \\
\hline $\begin{array}{l}\text { Geral } \\
\text { Overall }\end{array}$ & 45,47 & $0,68 \pm 20,96$ & $-59,79$ & 46,21 \\
\hline
\end{tabular}

Com o propósito de verificar como os reprodutores seriam classificados nos diferentes níveis de produção, foram calculadas as correlações de Spearman e de Pearson entre os valores genéticos dos reprodutores, obtidos por meio das equações de modelos mistos, em ambos níveis, e, ainda, a situação em que os rebanhos foram considerados conjuntamente (Tabela 5).

Quando os reprodutores são avaliados tomandose todos os rebanhos conjuntamente, verifica-se que há maior associação com rebanhos pertencentes ao nível de alta produção. Assim, novamente o resultado reforça que é esperado que a resposta à seleção seja também o resultado do número de filhas utilizadas na avaliação do reprodutor, bem como dos ambientes nos quais elas se expressaram e da distribuição destas filhas nestes ambientes.

Reprodutores cujas filhas manifestam suas produções em ambientes melhores podem ser beneficiados em um esquema de avaliação genética, o que compromete a confiabilidade dos resultados, mesmo em pequena escala. Correlações de ordem e produtomomento entre os valores genéticos dos reprodutores para a produção de leite maiores que 0,90 foram verificadas por Boldman \& Freeman (1990), Wiggans \& Van Raden (1991), Torres (1998) e Araújo (2000).

Tabela 5 - Correlações de Spearman (acima da diagonal) e de Pearson (abaixo da diagonal) entre os valores genéticos dos reprodutores, para as produções de leite e gordura nos níveis de produção alto e baixo e desconsiderando os níveis de produção (geral)

Table 5 - Spearman correlation (upper on diagonal) Pearson correlation (below on diagonal) between breeding values of sires to milk and fat yield in hight and low production levels and in analysis desconsidering the production levels (overall)

\begin{tabular}{|c|c|c|c|c|c|c|}
\hline \multirow[t]{2}{*}{$\begin{array}{l}\text { Níveis de produção } \\
\text { Production of level }\end{array}$} & \multicolumn{3}{|c|}{$\begin{array}{c}\text { Produção de leite } \\
\text { Milkyield }\end{array}$} & \multicolumn{3}{|c|}{$\begin{array}{c}\text { Produção de gordura } \\
\text { Fat yield }\end{array}$} \\
\hline & $\begin{array}{l}\text { Alto } \\
\text { Hight }\end{array}$ & $\begin{array}{l}\text { Baixo } \\
\text { Low }\end{array}$ & $\begin{array}{c}\text { Geral } \\
\text { Overall }\end{array}$ & $\begin{array}{l}\text { Alto } \\
\text { Hight }\end{array}$ & $\begin{array}{l}\text { Baixo } \\
\text { Low }\end{array}$ & $\begin{array}{c}\text { Geral } \\
\text { Overall }\end{array}$ \\
\hline Alto & 1,0000 & 0,9290 & 0,9819 & 1,0000 & 0,8723 & 0,9735 \\
\hline $\begin{array}{l}\text { High } \\
\text { Baixo }\end{array}$ & 0,9425 & 1,0000 & 0,9562 & 0,8922 & 1,0000 & 0,9237 \\
\hline $\begin{array}{l}\text { Gow } \\
\text { Overal }\end{array}$ & 0,9885 & 0,9603 & 1,0000 & 0,9832 & 0,9307 & 1,0000 \\
\hline
\end{tabular}

\section{R. Bras. Zootec., v.31, n.3, p.1343-1349, 2002 (suplemento)}




\section{Conclusões}

Estimativas de herdabilidade e correlação genética para a produção de leite revelaram que a heterogeneidade de variância presente é, principalmente, de natureza não genética, afetando, sobretudo, rebanhos de menores produções. Para a produção de gordura, os diferentes valores estimados para herdabilidade nos níveis de produção e o alto valor de correlação genética mostraram que a fonte de heterogeneidade de variância resulta, além de fatores ambientais, também de fatores genéticos.

Se a seleção de reprodutores é realizada por meio do desempenho de suas filhas, sem considerar essa variabilidade entre rebanhos, pode-se promover falhas na avaliação genética dos animais, visto que os grupos mais variáveis estão com maior número de animais. Como resultado, a seleção dos animais podem estar sendo realizada pelo melhor ambiente, $\mathrm{e}$ não pelo verdadeiro mérito genético dos animais.

\section{Agradecimento}

À Associação Brasileira de Criadores de Gado Pardo-Suíço, pelo fornecimento dos dados utilizados neste estudo.

\section{Literatura Citada}

ARAÚJO, C.V. Efeito da interação reprodutor $\mathbf{x}$ rebanho sobre a produção de leite na raça Holandesa. Viçosa, MG: Universidade Federal de Viçosa, 2000. 80p. Dissertação (Mestrado em Zootecnia) - Universidade Federal de Viçosa, 2000.

BOLDMAN, K.G.; FREEMAN, A.E. Adjustment for heterogeneity of variances by herd production level in dairy cow and sire evaluation. Journal of Dairy Science. v.73, n.2, p.503-512, 1990.

BOLDMAN, K.G.; KRIESE, L.A.; VAN VLECK, L.D. et al. A manual for use of MTDFREML: a set of programs to obtain estimates of variances and covariances (DRAFT). Lincoln: Department of Agriculture/Agricultural Research Service, $1995.120 \mathrm{p}$.

COSTA, C.N. Genetic relationships for milk and fat yields between Brazilian and United States Holstein cattle populations. Ithaca: Cornell University, 1998. 175p. Thesis (Doctor of Philosophy) - Cornell University, 1998.
COSTA, C.N. An investigation into heterogeneity of variance for milk and fat yields of Holstein cows in Brazilian herd environments. Genetic and Molecular Biology, v.22, n.3, p.375-381, 1990 .

DONG, M.C.; MAO, I.L. Heterogeneity of (co)variance and heritability in different levels of intra-herd milk production variance and of herd average. Journal of Dairy Science. v.73, n.3, p.843-851, 1990.

HILL, W.G.; EDWARDS, M.R.; AHMED, M.K.A. et al. Heritability of milk yield and composition at different levels and variability of production. Animal Production, v.36, n.1, p.59-68, 1983

JOHNSON, R.A.; WICHERN D.W. Applied multivariate statistical analysis. 4.ed. New Jersey: Prentice Hall, 1998. 816p.

LEE, K.J.; PARK, D.K. Heterogeneity of milk production levels in Korean dairy herds. In: WORLD CONGRESS ON GENETICS APLLIED TOLIVESTOCK PRODUCTION, 5., 1994, Ontário. Proceedings... Ontario: 1994, v.17, p.46-49.

RENNÓ, F.P. Aspectos produtivos da raça Pardo-Suíço no Brasil. Viçosa: Universidade Federal de Viçosa, 2001. 104p. Dissertação (Mestrado em Zootecnia) - Universidade Federal de Viçosa, 2001.

STANTON, T.L.; BLAKE, R.W.; QUASS, R.L. et al. Genotype by enviroment interaction for Holstein milk yield in Colombia, México and Porto Rico. Journal of Dairy Science, v.74, n.5, p.1700-1714, 1991.

SHORT, T.H.; BLAKE, R.W.; QUAAS, R.L. et al. Heterogeneous within-herd variance: 1 Genetic parameters for first and second lactations milk yield of grade Holtein cows. Journal of Dairy Science, v.73, n.11, p.3312-3320, 1990.

TORRES, R.A. Efeito da heterogeneidade de variância na avaliação genética de bovinos da raça Holandesa no Brasil. Belo Horizonte: Universidade Federal de Minas Gerais, 1998. 124 p. Tese (Doutorado em Ciência Animal) Universidade Federal de Minas Gerais, 1998.

TORRES, R.A.; BERGMANN, J.A.G.; COSTA, C.N. et al Heterogeneidade de variancia e avaliação genética de bovinos da raça Holandesa no Brasil. Revista Brasileira de Zootecnia, v.29, n.4, p.1050-1059, 2000.

WIGGANS, G.R.; VANRADEN, P.M. Method and effect of adjustment for heterogeneous variance. Journal of Dairy Science, v.74, n.12, p.4350-4357, 1991. 